\title{
Patients with depression display cytokine levels in serum and cerebrospinal fluid similar to patients with diffuse neurological symptoms without a defined diagnosis
}

\author{
This article was published in the following Dove Press journal: \\ Neuropsychiatric Disease and Treatment \\ 13 April 2016 \\ Number of times this article has been viewed
}

\author{
Knut A Hestad, ${ }^{1-3}$ Knut \\ Engedal, ${ }^{4}$ Jon Elling \\ Whist,' Pål Aukrust, ${ }^{5-9}$ \\ Per G Farup, ${ }^{1,10}$ Tom \\ Eirik Mollnes, ${ }^{9,11-13}$ Thor \\ Ueland $d^{5,7,9}$
}

'Department of Research, Innlandet Hospital Trust, Brumunddal,

${ }^{2}$ Department of Psychology, Faculty of Social Sciences and Technology Management, Norwegian University of Science and Technology (NTNU), Trondheim, ${ }^{3}$ Department of Public Health, Hedmark University College, Elverum, ${ }^{4}$ Norwegian Centre for Aging and Health, Vestfold Health Trust, Tønsberg, ${ }^{5}$ Research Institute of Internal Medicine, ${ }^{6}$ Section of Clinical Immunology and Infectious Diseases, ${ }^{7}$ Institute of Clinical Medicine, Oslo University Hospital Rikshospitalet, ${ }^{8}$ K.G. Jebsen IRC, University of Oslo, Oslo, ${ }^{9} \mathrm{~K} . \mathrm{G}$. Jebsen TREC, University of Tromsø, Troms $\varnothing,{ }^{10}$ Unit for Applied Clinical Research, Department of Cancer Research and Molecular Medicine, Faculty of Medicine, Norwegian University of Science and Technology, Trondheim, "Centre of Molecular Inflammation Research, Norwegian University of Science and Technology (NTNU), Trondheim, ${ }^{12}$ Department of Immunology, Oslo University Hospital Rikshospitalet, Oslo, ${ }^{13}$ Research Laboratory,

Nordland Hospital, Bodø, Norway

Correspondence: Knut A Hestad Department of Research, Innlandet Hospital Trust, Post Box 104, 238 I Brumunddal, Norway

Email knut.hestad@sykehuset-innlandet.no
Introduction: Several reports indicate that inflammation may play a role in depression and demonstrate enhanced systemic levels of inflammatory mediators. We hypothesized that 44 patients with a diagnosis of depression would present with a specific and different serum and cerebrospinal fluid (CSF) cytokine profile compared to 21 patients with diffuse neurological symptoms, of whom 15 had fatigue as a major symptom, but no change in emotional state.

Methods: The diagnoses of the patients with depression were according to the International Classification of Diseases, tenth edition (F32-34 spectra). Cytokine profiles in serum and CSF were determined by multiplex analysis, including 27 cytokines, chemokines, and growth factors.

Results: No differences could be found between the two groups studied regarding cytokine levels in serum or CSF except for serum interleukin (IL)-1 receptor antagonist that was lower in the depression group. There were only four high correlations $(>0.4)$ between serum and CSF levels of the cytokines, reflecting independent synthesis and turnover in these two compartments. In the control group, fatigue was associated with increased IL-1 receptor antagonist, IL-10, granulocyte-colony stimulating factor, and interferon- $\gamma$ (all $P<0.01$ ).

Conclusion: Patients with depression had a similar cytokine profile as nondepressive patients, both systemically and in CSF. Fatigue was associated with higher levels of some inflammatory markers in the control group. It is possible that the presence of fatigue in a large proportion of patients and controls could contribute to the lack of difference in cytokine levels between these two groups.

Keywords: depression, cytokines, chemokines, inflammation, fatigue, serum, CSF, multiplex analysis

\section{Introduction}

Depression is the most widespread mental disorder in the world as $>350$ million individuals suffer from the disorder worldwide. ${ }^{1}$ Three general mechanisms have been suggested to influence biomarkers related to depression, namely, through neurotransmitter signaling, the hypothalamic-pituitary-adrenal axis, and inflammation. Alterations in these interacting pathways also seem to play a pathogenic role in depressive disorders. As for inflammation, several lines of evidence support a role for activation of these pathways in the progression of depression. First, several studies have shown increased systemic levels of inflammatory cytokines and other inflammatory markers in patients with depression, ${ }^{2,3}$ and a recent gene expression study in a 
large population of patients with major depression disorder revealed a marked regulation of inflammatory and immunerelated pathways. ${ }^{4}$ Second, in patients receiving treatment with cytokines or other immune modulators, a considerable proportion may develop symptoms of severe depression. ${ }^{5-7}$ Finally, some anti-inflammatory drugs have been shown to exert antidepressive effects. ${ }^{8,9}$

Other disorders related to psychological disturbances such as chronic fatigue and fibromyalgia may also show signs of systemic inflammation even though they do not show any changed emotional state as in depression. ${ }^{10,11}$

Moreover, while several studies have examined plasma or serum levels of inflammatory markers in depressive disorders, data on these markers in cerebrospinal fluid (CSF) are scarce. ${ }^{3}$ Finally, cytokines and inflammatory mediators operate in network, and while several studies ${ }^{3}$ have examined some standard inflammatory cytokines such as tumor necrosis factor and interleukin (IL)-6 in these patients, fewer have simultaneously examined a wide range of inflammatory and anti-inflammatory markers reflecting the cytokine network.

We hypothesized that patients with a diagnosis of depression would present with a specific and different serum and CSF cytokine network profile compared to patients with diffuse neurological symptoms, but with no change in the emotional state.

\section{Materials and methods Patients and controls}

A group of depressed patients $(n=49)$ were recruited from inpatient wards and outpatient clinics of the mental health unit at Innlandet Hospital Trust in the eastern part of Norway. They were all older than 18 years and had a diagnosis of depression according to the research criteria of International Classification of Diseases, tenth edition (F32-34 spectra) and Diagnostic and Statistical Manual of Mental Disorders, fourth edition, text revision. Five of these patients were excluded because they were not able to give CSF sample for different reasons. The control group comprised 21 patients with neurological symptoms referred to the Department of Neurology at the same hospital for thorough investigations. They all had a careful neurological examination, including computed tomography/magnetic resonance imaging scans and spinal fluid examination. None of them had any neurological signs of disease, and neither did any of them have any laboratory findings indicating cerebral disease or disorder. Clinically, they had many of the same components as in depression, including tiredness, fatigue, lack of initiative, and a feeling that the body is not functioning, but without any overt signs of depression, ie, change in mood or emotional state. A medical history was recorded, a routine clinical examination was performed, and hematological and biochemical screening tests were carried out in all patients. None of the participants included in the study had any known comorbid disorders such as infection or inflammatory disorders that could influence the levels of cytokines. The data were collected between October 2010 and December 2013 by the same personnel and handled and stored at the hospital laboratory.

\section{Ethics}

The study was approved by the Norwegian Regional Committees for Medical and Health Research Ethics. Written informed consent was given by all participants before inclusion.

\section{Psychiatric and neuropsychological examination}

All patients were examined with the Montgomery-Åsberg Depression Rating Scale (MADRS; ${ }^{12}$ no depression 0-6, mild depression 7-19, moderate depression 20-34, and severe depression $>34$ ), the Beck Depression Inventory-II (BDI-II; ${ }^{13-15}$ minimal depression 0-13, mild depression 14-19, moderate depression 20-28, and severe depression 29-63), and the Mini-Mental Status Examination (MMSE) ${ }^{16}$ for cognitive functioning/dementia (score 0-30, normal $\geq 24$ ). The participants were asked about fatigue in the clinical interview, and questions about these symptoms are also one of the items on the BDI-II and MADRS.

\section{Collection of serum and CSF}

Peripheral blood was collected and allowed to clot for 30 minutes before centrifugation for 10 minutes at 2,000 $\times g$. CSF samples were obtained by a trained neurologist and centrifuged within 30 minutes at $2,000 \times g$ for 10 minutes. Serum and CSF were aliquoted and stored at $-70^{\circ} \mathrm{C}$ until analyses were performed. All blood samples were taken between 8.30 am and $10 \mathrm{am}$. The spinal tap was done before $12 \mathrm{pm}$, except for two depressed patients who did the procedure closer to $1 \mathrm{pm}$. None of the patients were fasting as a prerequisite before blood or CSF sampling.

\section{Multiplex analysis}

The samples were analyzed according to the instructions from the manufacturer on a Multiplex Analyzer using a multiplex cytokine assay (Bio-Plex Human Cytokine 27-Plex Panel; Bio-Rad Laboratories Inc., Hercules, CA, USA) containing the following ILs, chemokines, and growth factors: IL-1 $\beta$; IL-1 receptor antagonist (IL-1Ra); IL-2; IL-4; IL-5; IL-6; IL-7; IL-8 (CXCL8); IL-9; IL-10; IL-12 (p70); IL-13; IL-15; IL-17; 
Table 3 Comparison of cytokine cerebrospinal fluid levels $(\mathrm{pg} / \mathrm{mL})$ for the two study groups

\begin{tabular}{|c|c|c|c|}
\hline Cytokine & $\begin{array}{l}\text { Neurological group } \\
\text { (mean, SD), N=2 I }\end{array}$ & $\begin{array}{l}\text { Depression group } \\
\text { (mean, SD), N=44 }\end{array}$ & P-value* \\
\hline IL-I $\beta$ & $0.4(I . I)$ & $0.2(0.3)$ & 0.21 \\
\hline IL-IRa & $0.8(0.3)$ & $0.8(0.3)$ & 0.13 \\
\hline IL-2 & $0.7(0.4)$ & $0.5(0.4)$ & 0.30 \\
\hline IL-4 & $0.1(0.1)$ & $0.1(0.1)$ & 0.27 \\
\hline IL-5 & $0.2(0.1)$ & $0.3(0.4)$ & 0.65 \\
\hline IL-6 & $3.3(1.3)$ & $4.0(3.2)$ & 0.75 \\
\hline \multicolumn{4}{|l|}{ IL-7a } \\
\hline IL-8 & $36(13)$ & $38(15)$ & 0.49 \\
\hline IL-9 & $3.3(1.1)$ & $3.3(0.9)$ & 0.21 \\
\hline IL-IO & I.6 (0.4) & I.5 (0.3) & 0.40 \\
\hline \multicolumn{4}{|l|}{ IL- 12 p70a } \\
\hline IL-13 & $4.6(7.3)$ & $5.8(12)$ & 0.70 \\
\hline IL-I5 & $5.4(1.5)$ & $5.4(1.8)$ & 0.57 \\
\hline IL-I7A & $6.3(3.9)$ & $6.2(3.7)$ & 0.56 \\
\hline Eotaxin & $3.7(5.7)$ & $3.1(8.5)$ & 0.45 \\
\hline FGF-basic & $7.0(3.7)$ & $6.0(3.4)$ & 0.34 \\
\hline \multicolumn{4}{|l|}{ G-CSFa } \\
\hline GM-CSF & $45(7.7)$ & 45 (7.7) & 0.69 \\
\hline $\mathrm{IFN}-\gamma$ & $3.9(9.0)$ & $2.9(8.1)$ & 0.58 \\
\hline IP- 10 & 670 (399) & $693(424)$ & 0.50 \\
\hline MCP-I & $287(130)$ & $286(116)$ & 0.87 \\
\hline MIP-I $\alpha$ & $1.0(0.3)$ & I $(0.3)$ & 0.29 \\
\hline MIP-I $\beta$ & $17(6.6)$ & $14(4.7)$ & 0.06 \\
\hline PDGF-BB & $3.7(2.7)$ & $4.3(3.9)$ & 0.24 \\
\hline RANTES & $2.7(2.2)$ & $3.6(6.5)$ & 0.13 \\
\hline $\mathrm{TNF} \alpha$ & $1.6(0.9)$ & I.4 (0.8) & 0.29 \\
\hline VEGF & $15(5.5)$ & I5 (5.4) & 0.16 \\
\hline
\end{tabular}

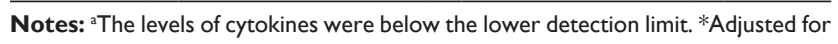
use of antipsychotic and antidepressive medication.

Abbreviations: IL, interleukin; IL-IRa, IL-I receptor antagonist; FGF, fibroblast growth factor; G-CSF, granulocyte-colony stimulating factor; GM-CSF, granulocyte macrophage CSF; IFN, interferon; IP- I0, IFN-inducible protein I0; MCP-I, monocyte chemoattractant protein I; MIP-I, macrophage inflammatory peptide I; PDGF-BB, platelet-derived growth factor-BB; RANTES, regulated on activation, normal T-cell expressed and secreted; TNF, tumor necrosis factor; VEGF, vascular endothelial growth factor.

with MADRS and BDI-II and serum cytokine levels. In CSF, severity of symptoms and IL-15 (BDI-II: $\rho=-0.37$, $P=0.01$; MADRS: $\rho=-0.47, P=0.001$ ) and MCP-1 (BDI-II: $\rho=-0.54, P<0.001)$ were correlated. In CSF of the controls, IL-2 levels were correlated with the severity of symptoms (BDI-II [IL-2]: $\rho=-0.63, P=0.004)$. On BDI-II, there is partly an overlap between depression symptoms and symptoms related to physical and psychological functions such as change in appetite, fatigue and tiredness, and concentration difficulty, which may also give elevated scores on MADRS or BDI-II in the control group even though the patients are not depressed.

\section{Fatigue symptoms and cytokine levels}

There was an overlap in symptoms between the two groups studied, especially related to fatigue. In the depressed group,
42 of the 44 patients reported to experience fatigue to be part of the depression disorder. In the control group, 15 of the 21 patients had fatigue.

These 15 with fatigue symptoms had significantly higher serum levels of IL-1Ra $(P=0.010)$, IL-10 ( $P=0.004)$, G-CSF $(P=0.006)$, and IFN- $\gamma(P=0.004)$ than those without fatigue symptoms $(\mathrm{n}=6)$. In contrast, $\mathrm{CSF}$ values did not discriminate between those with fatigue and those without. As fatigue most probably is a part of the phenotype of depression, being present in 42 of the 44 patients with depression, we were precluded from doing a similar analysis in depressive patients.

\section{Correlations between cytokine levels in serum and CSF}

Within the control group, there was high correlation between eotaxin in serum and CSF $(\rho=0.63, P=0.002)$. For the patients with depression, high correlations were seen for eotaxin ( $\rho=0.44, P=0.003)$, IP-10 ( $\rho=0.46, P=0.002)$, and MIP-1 $\beta(\rho=0.46, P=0.002)$. Otherwise, correlations were lower than 0.4 .

\section{Discussion}

In contrast to our hypothesis that patients with major depression would present with a specific and different serum and CSF cytokine network profile compared to patients with diffuse neurological symptoms, we observed no differences in the levels of a wide range of cytokines in either serum or CSF between the two study groups, except for marginally lower levels of IL-1Ra in depressive patients. Furthermore, except for a few chemokines, relatively few correlations were found between serum and CSF suggesting limited interplay between these compartments in these individuals, with no differences between depressive patients and controls. However, it must be emphasized that it is difficult to draw firm conclusions from such a small sample size as in this study. Also, a limitation of the study is that some degree of intraindividual variability occurs in the methods used in the study. Therefore, repeated measurements over time are the best way to control such variations. Nevertheless, if it is difficult to obtain approval for one CSF sample, it would be very complicated to get multiple samples from the same individual. However, one cannot exclude that some physiological variability adds a layer of uncertainty to the pathological variation when measuring a single time point.

Based on the growing body of evidence demonstrating elevated systemic levels of proinflammatory cytokines and several chemokines in blood of depressed patients, ${ }^{3}$ it is surprising that we found no associations between systemic 
markers and severity of depressive symptoms in the serum of the patient group. However, whereas most previous studies (e.g. Hestad et al 2009) ${ }^{17}$ have compared inflammatory serum markers in patients with depression and healthy controls, we in the present study examined these markers in both serum and CSF and included a control group with a certain degree of overlap in symptoms, especially related to fatigue, but without a depressive disorder. Several studies have demonstrated that depression and chronic fatigue and fibromyalgia share aberrations in inflammatory pathways, ${ }^{18}$ and it is not inconceivable that this could have contributed to the lack of differences in cytokine levels between the two groups of individuals in the present study. It is possible that depression cannot be distinguished from patients who suffer from fatigue with respect to systemic or CSF values of inflammatory markers because a large proportion of the depressive patients also have similar symptoms. However, it is suggested that possible inflammatory processes seen in depression might be closely linked with multiple neurodegenerative pathways. ${ }^{19,20}$ Advanced aging and a number of risk factors such as obesity, hypertension, diabetes, and atherosclerosis could act as "silent contributors" promoting a chronic proinflammatory state. Brain reserves may have neuroprotective effects against late life depression. ${ }^{21}$ In our project, the study population was relatively young with a mean age of $\sim 45$ and no degenerative signs of sickness. It may be that a higher age and more comorbidity would have contributed to a higher level of inflammation among the depressed participants.

A few studies have examined the cytokine level in CSF in patients with depression. Evidence has shown increased levels of IL-1 $\beta^{22}$ and divergent results for IL- $6^{22,23}$ and MCP- $1 .{ }^{24}$ The last study was related to medication with IFN- $\alpha$, while Lindqvist et $\mathrm{al}^{23}$ studied suicide attempters and normal controls and Levine et $\mathrm{al}^{22}$ used patients who were evaluated for headache or suspended meningitis as controls. However, no studies have compared levels in CSF with systemic levels of inflammatory markers. Chemokines may enhance permeability of the blood-brain barrier and increase neuroinflammation by promoting infiltration of inflammatory cells. ${ }^{25} \mathrm{In}$ general, we found few correlations $>0.4$ between the two compartments. However, our finding that eotaxin, IP-10, and MIP-1 $\beta$ showed a high correlation between the two compartments could suggest a potential role for these chemokines in the regulation of neuroinflammation.

Strengths of our study include the case-control design where all the patients came from the same county, were examined at the same hospital, and had an overlap in some but not in all depressive symptoms. The multiplex assay gave us the opportunity to evaluate the inflammatory reaction broadly in both serum and CSF with an acceptable coefficient of variation. However, as there are no universal or official reference range values for the analyses used in the present study, the lack of a normal healthy population group is a limitation of the present study with respect to comparison of the levels of the cytokines in these two groups with a reference population. A limitation of the study is that some degree of intraindividual variability occurs that repeated measurements over time could control for. However, this is not easily done regarding CSF where the method is somewhat more difficult than taking a blood sample. Finally, the subgroup analysis comparing the levels of inflammatory markers in controls with and without fatigue had small groups, and caution is needed when interpreting these results.

\section{Conclusion}

In conclusion, compared to patients with diffuse neurological symptoms with fatigue as a major symptom, but with no change in the emotional state, patients with major depression did not display any major alterations in systemic or CSF levels of cytokines and growth factors. The general lack of correlation between serum and CSF suggests limited interactions between the two compartments for the mediators investigated. Fatigue was associated with higher levels of some inflammatory markers, and it is possible that the presence of this symptom in a large proportion of patients and controls could contribute to the lack of difference in cytokine levels between these two groups.

\section{Acknowledgments}

The authors thank Gunnar Børre Thoresen and his team at Innlandet Hospital Trust and Christa Kristiansen for data collection. The authors also thank the study participants and the participating institutions for their cooperation, and Lillehammer University College and Innlandet Hospital Trust for funding.

\section{Disclosure}

The authors report no conflicts of interest in this work.

\section{References}

1. WHO [webpage on the Internet]. Depression: Fact Sheet $\mathrm{N}^{\circ} 369$ Available from: http:/www.who.int/mediacentre/factsheets/fs369/en/. Accessed January 29, 2016.

2. Poon DC, Ho YS, Chiu K, Wong HL, Chang RC. Sickness: from the focus on cytokines, prostaglandins, and complement factors to the perspectives of neurons. Neurosci Biobehav Rev. 2015;57:30-45.

3. Young JJ, Bruno D, Pomara N. A review of the relationship between proinflammatory cytokines and major depressive disorder. J Affect Disord. 2014;169:15-20.

4. Jansen R, Penninx BW, Madar V, et al. Gene expression in major depressive disorder. Mol Psychiatry. Epub 2015 May 26. 
5. Capuron L, Gumnick JF, Musselman DL, et al. Neurobehavioral effects of interferon-alpha in cancer patients: phenomenology and paroxetine responsiveness of symptom dimensions. Neuropsychopharmacology. 2002;26(5):643-652.

6. Capuron L, Ravaud A, Neveu PJ, Miller AH, Maes M, Dantzer R. Association between decreased serum tryptophan concentrations and depressive symptoms in cancer patients undergoing cytokine therapy. Mol Psychiatry. 2002;7(5):468-473.

7. Dantzer R, Wollman EE, Vitkovic L, Yirmiya R. Cytokines, stress, and depression. Conclusions and perspectives. Adv Exp Med Biol. 1999;461:317-329.

8. Watson S, Gallagher P, Ritchie JC, Ferrier IN, Young AH. Hypothalamicpituitary-adrenal axis function in patients with bipolar disorder. $\mathrm{Br} J$ Psychiatry. 2004;184:496-502.

9. Kohler O, Benros ME, Nordentoft M, et al. Effect of anti-inflammatory treatment on depression, depressive symptoms, and adverse effects: a systematic review and meta-analysis of randomized clinical trials. JAMA Psychiatry. 2014;71(12):1381-1391.

10. Romano GF, Tomassi S, Russell A, Mondelli V, Pariante CM. Fibromyalgia and chronic fatigue: the underlying biology and related theoretical issues. Adv Psychosom Med. 2015;34:61-77.

11. Maes M, Twisk FN, Kubera M, Ringel K. Evidence for inflammation and activation of cell-mediated immunity in Myalgic Encephalomyelitis/ Chronic Fatigue Syndrome (ME/CFS): increased interleukin-1, tumor necrosis factor-alpha, PMN-elastase, lysozyme and neopterin. J Affect Disord. 2012;136(3):933-939.

12. Montgomery SA, Asberg M. A new depression scale designed to be sensitive to change. Br J Psychiatry. 1979;134:382-389.

13. Beck AT, Steer RA, Ball R, Ranieri W. Comparison of Beck Depression Inventories-IA and -II in psychiatric outpatients. J Pers Assess. 1996;67(3):588-597.

14. Steer RA, Ball R, Ranieri WF, Beck AT. Further evidence for the construct validity of the Beck depression Inventory-II with psychiatric outpatients. Psychol Rep. 1997;80(2):443-446.

15. Steer RA, Ball R, Ranieri WF, Beck AT. Dimensions of the Beck Depression Inventory-II in clinically depressed outpatients. J Clin Psychol. 1999;55(1):117-128.
16. Folstein MF, Folstein SE, McHugh PR. "Mini-mental state". A practical method for grading the cognitive state of patients for the clinician. J Psychiatr Res. 1975;12(3):189-198.

17. Hestad K, Aukrust P, Tønseth S, Reitan SK. Depression has a Strong Relationship to Alterations in the Immune, Endocrine and Neural System. Current psychiatry reviews. 2009;5(4):287-297.

18. Maes M. An intriguing and hitherto unexplained co-occurrence: depression and chronic fatigue syndrome are manifestations of shared inflammatory, oxidative and nitrosative (IO\&NS) pathways. Prog Neuropsychopharmacol Biol Psychiatry. 2011;35(3):784-794.

19. Sandu RE, Buga AM, Uzoni A, Petcu EB, Popa-Wagner A. Neuroinflammation and comorbidities are frequently ignored factors in CNS pathology. Neural Regen Res. 2015;10(9):1349-1355.

20. Popa-Wagner A, Buga AM, Tica AA, Albu CV. Perfusion deficits, inflammation and aging precipitate depressive behaviour. Biogerontology. 2014;15(5):439-448.

21. Freret T, Gaudreau P, Schumann-Bard P, Billard JM, Popa-Wagner A. Mechanisms underlying the neuroprotective effect of brain reserve against late life depression. JNeural Transm (Vienna). 2015;122(suppl 1): S55-S61.

22. Levine J, Barak Y, Chengappa KN, Rapoport A, Rebey M, Barak V. Cerebrospinal cytokine levels in patients with acute depression. $\mathrm{Neu}$ ropsychobiology. 1999;40(4):171-176.

23. Lindqvist D, Janelidze $S$, Hagell $P$, et al. Interleukin-6 is elevated in the cerebrospinal fluid of suicide attempters and related to symptom severity. Biol Psychiatry. 2009;66(3):287-292.

24. Raison CL, Borisov AS, Majer M, et al. Activation of central nervous system inflammatory pathways by interferon-alpha: relationship to monoamines and depression. Biol Psychiatry. 2009;65(4):296-303.

25. Takeshita Y, Ransohoff RM. Inflammatory cell trafficking across the blood-brain barrier: chemokine regulation and in vitro models. Immunol Rev. 2012;248(1):228-239.
Neuropsychiatric Disease and Treatment

\section{Publish your work in this journal}

Neuropsychiatric Disease and Treatment is an international, peerreviewed journal of clinical therapeutics and pharmacology focusing on concise rapid reporting of clinical or pre-clinical studies on a range of neuropsychiatric and neurological disorders. This journal is indexed on PubMed Central, the 'PsycINFO' database and CAS,

\section{Dovepress}

and is the official journal of The International Neuropsychiatric Association (INA). The manuscript management system is completely online and includes a very quick and fair peer-review system, which is all easy to use. Visit http://www.dovepress.com/testimonials.php to read real quotes from published authors. 